\title{
Evapotranspiração e coeficientes de cultivo da beterraba orgânica sob cobertura morta de leguminosa e gramínea
}

\author{
Dionízio H Oliveira Neto; Daniel F de Carvalho; Leonardo DB da Silva; José Guilherme M Guerra; Mar- \\ cos B Ceddia \\ UFRRJ-Dep ${ }^{\text {to. }}$. Engenharia, Instituto de Tecnologia, BR 465, km 47, 23890-000 Seropédica-RJ; dionizioneto@ufrrj.br; carvalho@ufrrj.br
}

\section{RESUMO}

As práticas agrícolas que maximizam a produtividade e o uso da água são de vital importância para a agricultura. Assim, foram testados três tipos de manejo do solo com objetivo de determinar a evapotranspiração (ETc) e os coeficientes de cultivo (kc) da beterraba. Os tipos de manejo foram a utilização de coberturas mortas vegetais, denominadas capim cameroon (Pennisetum purpureum), gliricídia (Gliricidia sepium) e solo sem cobertura morta em área experimental do SIPA (Sistema Integrado de Produção Orgânica) localizado em Seropédica, Brasil. A lâmina de irrigação foi estimada com base no balanço de água no solo, cujo monitoramento foi realizado com a técnica da TDR. As ETc acumuladas para a cultura da beterraba foram 59,41; 55,31 e 119,62 mm, respectivamente, para capim cameroon, gliricídia e solo sem cobertura morta. A evapotranspiração de referência (ETo) foi obtida por meio do modelo de Penamn-Monteith. Os valores médios de kc obtidos para as fases inicial, média e final de desenvolvimento foram de 0,$39 ; 0,42$ e 1,02; 0,79; 0,76 e 1,18; e 0,$56 ; 0,61$ e 0,84 , respectivamente, para capim cameroon, gliricídia e solo sem cobertura morta. O uso da cobertura do solo com gramínea ou leguminosa minimizou de forma expressiva a demanda hídrica da cultura da beterraba (Beta vulgaris).

Palavras-chave: Beta vulgaris, Gliricidia sepium, Pennisetum purpureum, TDR, kc e mulching orgânico.

\begin{abstract}
Evapotranspiration and crop coefficient of beet in organic mulch of grass and legume

Agricultural practices that maximize productivity and water use are of vital importance to farming. Thus, three different soil managements were used in order to determine the evapotranspiration (ETc) and crop coefficients (kc) of beet in the experimental area of the Universidade Federal Rural do Rio de Janeiro, located in Seropédica, Rio de Janeiro State, Brazil. The used managements consisted of cameroon (Pennisetum purpureum), gliricídia (Gliricidia sepium) and bare soil. The irrigation depth was estimated based on the soil water balance in order to restore the amount of water until soil moisture corresponded to field capacity, monitored by a TDR technique. Reference evapotranspiration (ETo) was calculated using the Penamn-Monteith model. The accumulated ETc were 59.41, 55.31 and $119.62 \mathrm{~mm}$, respectively, for cameroon, gliricídia and bare soil. The values of $\mathrm{kc}$ obtained for the initial, middle and end developmental stages were $0.39,0.42$ and $1.02,0.79,0.76$ and 1.18 and $0.56,0.61$ and 0.84 , respectively, for cameroon, gliricídia and bare soil. The use of soil cover with grass or legume minimized significantly the water demand by beet crop.
\end{abstract}

Keywords: Beta vulgaris, Gliricidia sepium, Pennisetum purpureum, TDR, kc and organic mulching.

(Recebido para publicação em 22 de dezembro de 2009; aceito em 20 de junho de 2011)

(Received on December 22, 2009; accepted on June 20, 2011)

$\mathrm{O}$ plantio e o consumo de beterraba (Beta vulgaris) são amplamente difundidos entre produtores e consumidores de hortaliças do estado do Rio de Janeiro. Os fatores limitantes ao cultivo da beterraba são, segundo Filgueira (2000), a temperatura e pluviosidade elevadas, pois favorecem as ocorrências de doenças, formações de anéis de coloração clara no interior da raiz tuberosa e raízes lenhosas ou fibrosas.

Na região metropolitana do Rio de Janeiro cultiva-se a beterraba no inverno, coincidindo sua produção com a época de maior disponibilidade do produto no estado segundo calendário de comercialização da CEASA.
De acordo com Medeiros et al. (2006), a busca por melhores condições ambientais e a necessidade de melhorar a produção em quantidade e qualidade, demanda a adaptação de novas tecnologias de interesse e oferta de suporte às culturas, destacando-se dentre elas, o uso de cobertura morta vegetal no solo. Além da reduzida evapotranspiração sob cobertura morta no solo (Allen et al., 1998), outro efeito benéfico da cobertura morta está na oferta de nutrientes, os quais são disponibilizados durante o processo de decomposição (Aita \& Giacomini, 2003). Como exemplos, destacam-se as espécies vegetais leguminosas e em particular a gliricídia
(Gliricidia sepium). Esta espécie arbórea detém relevantes atributos como rusticidade e tolerância a podas drásticas sazonais, com acelerada e total regeneração da copa, que é particularmente rica em nitrogênio (Almeida, 2007; Almeida et al., 2008).

Tendo em vista a limitada disponibilidade espacial e temporal dos recursos hídricos, a implantação de sistemas de irrigação vem se tornando restrita em diferentes partes do mundo. Para isso, o conhecimento das reais necessidades hídricas das culturas é fundamental, sendo o efeito do estresse hídrico no crescimento e produtividade das culturas dependente da sua tolerância, resis- 
tência ou período de desenvolvimento mais sensível à falta d'água (Kadayifci et al., 2005).

O conhecimento do coeficiente de cultivo (kc) permite a estimativa das necessidades hídricas de uma cultura, sendo fundamental para o manejo da irrigação e, consequentemente, para a maximização do uso da água. Para beterraba de mesa, Allen et al. (1998) apresentam valores de kc de 0,50; 1,05 e 0,95 , nas fases inicial, média e final, respectivamente.

$\mathrm{Na}$ estimativa de kc para uma dada localidade, a evapotranspiração de referência (ETo) constitui um importante parâmetro, sendo o método de Penamn-Monteith o método padrão da FAO (Allen et al., 1998). A evapotranspiração é influenciada pela energia disponível na superfície, pelo gradiente de pressão de vapor entre a superfície e atmosfera e pela resistência às transferências de vapor (Souza Filho et al., 2005). Andrade et al. (2002) comentam que a palhada na superfície do solo altera as relações evapotranspirométricas, pois afeta a evaporação de água do solo, reduzindo assim a taxa de evapotranspiração das culturas. Esse fato propicia aumento do intervalo entre irrigações ou mesmo reduz as lâminas de irrigação a serem aplicadas, contribuindo para um aumento na eficiência do uso da água (Carvalho et al., 2011).

Tendo em vista a importância de se conhecer a real necessidade hídrica da cultura em diferentes tipos de manejo do solo, desenvolveu-se este trabalho com o objetivo de determinar a evapotranspiração e os coeficientes de cultivo da cultura da beterraba, em Seropédica, região metropolitana do estado do Rio de Janeiro.

\section{MATERIAL E MÉTODOS}

O experimento foi conduzido no SIPA (Sistema Integrado de Produção Agroecológica), localizado no município de Seropédica-RJ (2246'S; $43^{\circ} 41^{\prime} \mathrm{W}$; $32 \mathrm{~m}$ de altitude). De acordo com Carvalho et al. (2007), o clima da região é classificado como Aw segundo Köppen, com chuvas no verão e temperaturas elevadas, e um inverno seco com temperaturas amenas. As chuvas se concentram no período de novembro a março, com precipitação anual média de $1.213 \mathrm{~mm}$ e temperatura média anual de $24,5^{\circ} \mathrm{C}$.

O solo foi classificado como Argissolo Vermelho Amarelo e segundo Villela (2007), a área experimental está compreendida entre as regiões que apresentam área com restrição potencial por disponibilidade de ar às raízes (20-30\%) nas profundidades analisadas $(0-30 \mathrm{~cm})$.

A área foi preparada com uma aração e duas gradagens e, em seguida, os canteiros com 1,0 m de largura e 0,2 de altura foram levantados mecanicamente. A adubação orgânica foi realizada 5 dias antes do transplantio (23 de junho de 2008) utilizando esterco bovino curtido na dosagem de $25 \mathrm{t} \mathrm{ha}^{-1}$, segundo recomendação de De-Polli \& Almeida (1988).

Os experimentos constaram de diferentes tipos de manejo do solo: solo sem cobertura morta vegetal, solo com cobertura morta de leguminosa (Gliricidia sepium, gliricídia) e com cobertura morta de gramínea (Pennisetum purpureum, capim cameroon), sendo ambos picados e secos à sombra.

As mudas de beterraba cv. Early Wonder Tall Top foram produzidas em bandejas na casa-de-vegetação (Guimarães et al., 2002), abastecidas com substrato à base da vermicompostagem de esterco bovino, e transplantadas no espaçamento de $25 \times 15 \mathrm{~cm}$.

Durante os 25 dias de estabelecimento da cultura no campo, foram realizadas irrigações diárias utilizando sistema de irrigação por aspersão, que apresentava intensidade de precipitação média de $6,8 \mathrm{~mm} / \mathrm{h}$ e coeficiente de uniformidade de Christiassem (CUC) de 96\%. As lâminas aplicadas correspondiam a $100 \%$ da ETc, sendo esta estimada pelo produto da evapotranspiração de referência (ETo) pelo kc inicial de 0,50 (Allen et al., 1998). A estimativa da ETo foi realizada por meio do modelo de Penamn-Monteith FAO-56 (Allen et al., 1998), a partir de dados meteorológicos coletados em um estação automática localizada ao lado da área experimental.

As coberturas mortas de capim cameroon e gliricídia foram colocadas sobre os canteiros após o período de estabelecimento da cultura no campo, na quantidade de $2,5 \mathrm{~kg} / \mathrm{m}^{2}$, equivalendo à disposição de uma camada com espessura de $5 \mathrm{~cm}$, convertida em $\mathrm{kg}$ / $\mathrm{m}^{2}$, de acordo com a densidade do material picado e seco à sombra (Oliveira et al., 2008).

A partir daí, foi utilizado o sistema de irrigação por gotejamento, composto por gotejadores externos auto-reguláveis, com vazão de 7,8 L h ${ }^{-1}$. A lâmina de irrigação, aplicada diariamente, era calculada com base no monitoramento da umidade do solo, realizado pela técnica da TDR (Time Domain Reflectometry) (Topp et al., 1980). Em cada tipo de cobertura, 3 parcelas experimentais receberam sensores que foram instalados na posição horizontal, nas profundidades de 10 e $20 \mathrm{~cm}$. Inicialmente foi realizado um processo de calibração da TDR que consistiu na obtenção de leituras diárias da constante dielétrica aparente do solo (ka) e da umidade volumétrica $(\theta)$ correspondente, possibilitando obter, para as profundidades de $0-10$ e $10-20 \mathrm{~cm}$, equações de regressão lineares. Esse procedimento permitiu também determinar, in situ, os valores de $\theta$ correspondente à capacidade de campo, a qual serviu de referência para a reposição de água ao solo via irrigação. As equações de calibração obtidas foram: $\theta_{0-10}=$ $0,0199 \mathrm{ka}+0,0051 \mathrm{e}, \theta_{10-20}=0,0253$ $\mathrm{ka}-0,0874$, respectivamente, para as profundidades de 0-0,10 e 0,10-0,20 m. A ETc foi calculada utilizando o método do balanço de água no solo (Herman, 1985), expresso por: $\mathrm{ET}_{\mathrm{c}}=\mathrm{I}+\mathrm{Pe} \pm \Delta \mathrm{SW}$, em que I é a lâmina de irrigação aplicada (mm), Pe é a precipitação efetiva (mm) e $\Delta \mathrm{SW}$ é a variação do conteúdo de água armazenado no perfil (mm). Conforme Erdem et al. (2006), pelo fato da quantidade de água aplicada por irrigação ter sido suficiente apenas para elevar a umidade do solo à capacidade de campo, a lâmina correspondente à percolação profunda foi desprezada.

Outras variáveis do balanço hídrico como escoamento superficial, ascensão capilar e fluxos subsuperficiais de entrada e saída foram também considerados nulos, tendo em vista as características da área e do sistema de irrigação utilizado (Garcia y Garcia et al., 2009). A Pe foi considerada como sendo a lâmina de 
água precipitada $(\mathrm{mm})$ que proporcionou alteração na umidade do solo e que foi efetivamente disponibilizada para a cultura. Durante o manejo da irrigação, os valores de $\theta$ eram obtidos diariamente nas três parcelas-controle após as leituras da ka e, para cada condição de cobertura do solo, eram calculadas as lâminas de irrigação, considerando uma eficiência de aplicação $(\mathrm{Ea}) \mathrm{de}$ $90 \%$. Neste cálculo foi utilizada uma porcentagem de área molhada (PAM) de $50 \%$, que corresponde a um coeficiente de localização $\left(\mathrm{k}_{\mathrm{L}}\right)$ de 0,6 , utilizando a curva de Keller \& Bliesner (1990) apresentada por Mantovani et al.(2007).

Para as três condições de cobertura do solo, os valores de kc foram determinados diretamente por meio da razão entre a ETc e a ETo. Posteriormente, os valores médios de kc foram estimados para cada fase de cultivo da cultura da beterraba, sendo as mesmas definidas como: estádio $\mathrm{I}=$ até $15 \%$ da cobertura do solo (30 dias); estádio $\mathrm{II}=$ final do estádio I até $90 \%$ de cobertura do solo (24 dias) e estádio III= final do estádio II até a colheita (21 dias) (Costa et al., 2008). Os estádios de desenvolvimento preconizados por Doorenbos \& Kassam (1979) para o cultivo da beterraba açucareira, é em média, 70 dias maior que para a beterraba de mesa.

\section{RESULTADOS E DISCUSSÃO}

A precipitação, a temperatura média do ar e a ETo ocorridas durante o ciclo da cultura estão apresentadas na Tabela 1. Durante o período de cultivo, a chuva acumulada foi de $147 \mathrm{~mm}$, com o maior evento ocorrendo no dia $10 \mathrm{de}$ agosto $(58 \mathrm{~mm})$. As irrigações totais necessárias (ITN) para os três tipos de manejos do solo foram de 66, 79 e 136 $\mathrm{mm}$, e a chuva efetiva 9,$6 ; 9,9 ; 14,9 \mathrm{~mm}$; respectivamente, para cobertura morta com capim cameroon, gliricídia e na ausência de cobertura.

As variações dos perfis de umidade do solo $(\theta)$ para os três tipos de manejo são apresentadas na Figura 1. A linha pontilhada representa o valor de $\theta$ correspondente à capacidade de campo e que serviu de referência para reposição de $100 \%$ da ETc. A demanda por água para cada tipo de cobertura do solo foi

Tabela 1. Dados climatológicos agrupados em intervalos de 5 dias durante o período experimental de aplicação dos tratamentos (climatological data grouped in intervals of 5 days during the experimental period of treatment application). Seropédica, SIPA, 2008.

\begin{tabular}{lccccc}
\hline $\begin{array}{l}\text { Período } \\
(\mathbf{5} \text { dias })\end{array}$ & $\begin{array}{c}\text { Chuva } \\
(\mathbf{m m})\end{array}$ & $\begin{array}{c}\text { Rad } \\
\left(\mathbf{k J} / \mathbf{m}^{2}\right)\end{array}$ & $\begin{array}{c}\text { UR } \\
(\mathbf{\%})\end{array}$ & $\begin{array}{c}\text { Tmed } \\
\left({ }^{\mathbf{C}} \mathbf{C}\right)\end{array}$ & $\begin{array}{c}\text { ETo-PM } \\
(\mathbf{m m})\end{array}$ \\
\hline $17 / 7$ & 0,0 & 624,52 & 74 & 18,5 & 15,11 \\
$22 / 7$ & 0,0 & 601,61 & 70 & 20,8 & 15,48 \\
$27 / 7$ & 2,0 & 605,30 & 77 & 20,5 & 13,99 \\
$1 / 8$ & 0,0 & 653,17 & 68 & 22,0 & 15,45 \\
$6 / 8$ & 2,2 & 418,42 & 80 & 21,8 & 9,52 \\
$11 / 8$ & 66,5 & 615,80 & 84 & 21,6 & 5,54 \\
$16 / 8$ & 0,0 & 701,29 & 69 & 23,7 & 19,36 \\
$21 / 8$ & 0,0 & 732,52 & 62 & 23,7 & 18,11 \\
$26 / 8$ & 30,0 & 568,48 & 77 & 22,4 & 14,28 \\
$31 / 8$ & 10,0 & 526,21 & 75 & 19,4 & 9,21 \\
$5 / 9$ & 0,0 & 797,16 & 70 & 23,4 & 17,49 \\
$10 / 9$ & 7,1 & 688,35 & 80 & 22,6 & 10,57 \\
\hline
\end{tabular}

$\mathrm{Rad}=$ radiação global; $\mathrm{UR}=$ umidade relativa média; $\mathrm{ETo}-\mathrm{PM}=$ evapotranspiração de referência acumulada por Penman-Montieth ( $\mathrm{rad}=$ global radiation; $\mathrm{UR}=$ average relative humidity; ETo-PM= Penman-Monteith accumulated reference evapotranspiration).

Tabela 2. Valores de coeficiente de cultura $(\mathrm{kc})$ na área experimental em diferentes estádios do ciclo da beterraba em diferentes condições de cobertura do solo - Pennisetum purpureum cv. Cameroon, Gliricidia sepium (Gliricídia) e solo sem cobertura (crop coefficients (kc) in the experimental area at different stages of the cycle of beet under different soil cover - Pennisetum purpureum cv. Cameroon, Gliricidia sepium (Gliricidia) and soil without mulch). Seropédica, SIPA, 2008.

\begin{tabular}{|c|c|c|c|}
\hline \multirow[b]{2}{*}{ Cobertura do solo } & \multicolumn{3}{|c|}{ Estádios de desenvolvimento } \\
\hline & $\begin{array}{c}\text { Estádio I } \\
\text { (inicial) } 30 \text { dias }^{1}\end{array}$ & $\begin{array}{c}\text { Estádio II } \\
\text { (médio) } 24 \text { dias }\end{array}$ & $\begin{array}{c}\text { Estádio III } \\
\text { (final) } 21 \text { dias }\end{array}$ \\
\hline capim cameroon & 0,39 & 0,79 & 0,56 \\
\hline gliricídia & 0,42 & 0,76 & 0,61 \\
\hline sem cobertura & 1,02 & 1,18 & 0,84 \\
\hline boletim FAO $56^{(2)}$ & 0,50 & 1,05 & 0,95 \\
\hline
\end{tabular}

${ }^{1}$ Período em dias apresentados por Costa et al. (2008) (period in days presented by Costa et al. (2008)); ${ }^{2}$ Allen et al. (1998).

alterada em resposta às diferentes perdas por evapotranspiração. $\mathrm{Na}$ condição de solo sem cobertura, as aplicações diárias com maiores lâminas de irrigação, comparativamente, favoreceram a ocorrência de elevadas taxas de evapotranspiração na camada de $0-10 \mathrm{~cm}$, com maior acúmulo de água na camada de10-20 cm. Isso aconteceu em virtude do solo da área experimental apresentar maior porosidade na camada superficial encanteirada e por possuir características de um perfil $\mathrm{B}_{\mathrm{t}}$ argiloso abaixo da camada de $20 \mathrm{~cm}$. As oscilações de umidade na referida Figura coincidem com os eventos de precipitação ocorridos no período de monitoramento. Ao final do ciclo de cultivo, os valores de ETc para a cultura da beterraba acumularam 59,41; 55,31 e $119,62 \mathrm{~mm}$, respectivamente, para as condições de cobertura com capim cameroon, palhada de gliricídia e de solo sem cobertura morta.

A evapotranspiração de referência (ETo) acumulada foi de 145,14 mm. Durante os seis primeiros dias após período de estabelecimento da cultura, apenas o experimento sem cobertura demandou água suplementar (Figura 2). Ainda, nota-se que a ETc, a partir do dia 33, apresentou tendência em assumir valores superiores aos de ETo no experi- 


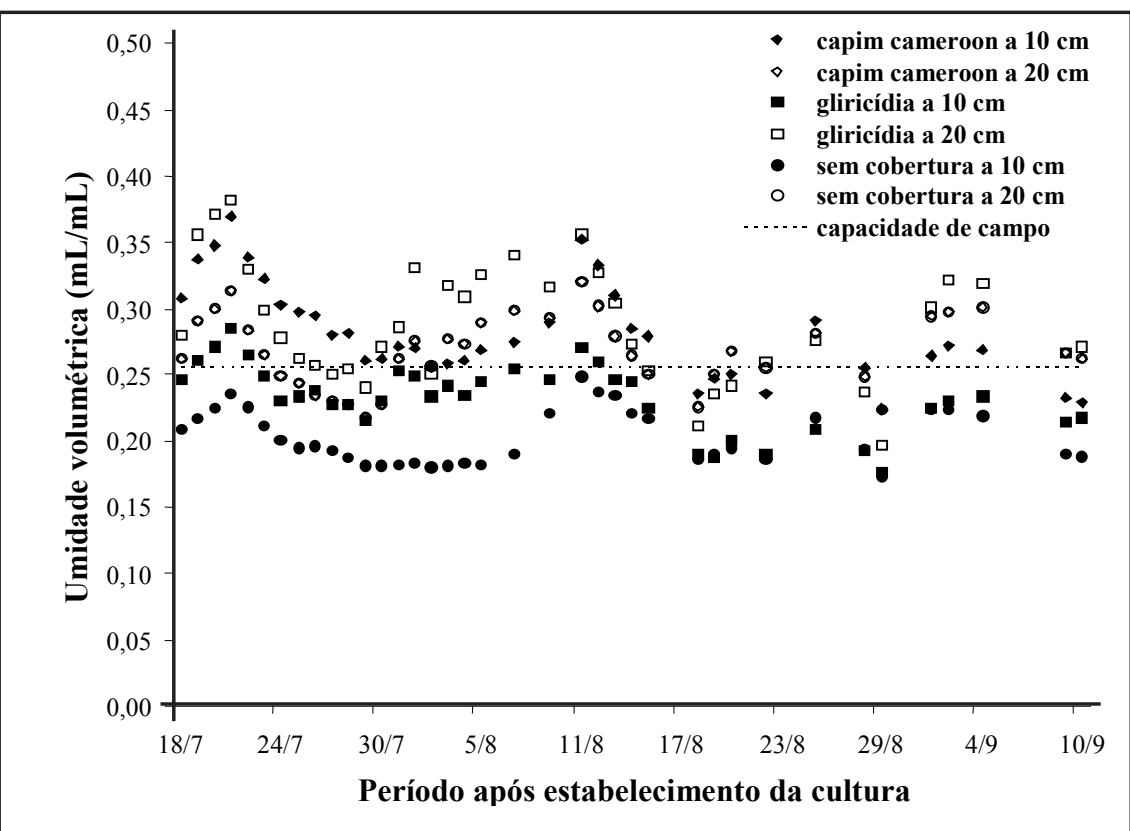

Figura 1. Valores de umidade do solo na área experimental (profundidades de 10 e $20 \mathrm{~cm}$ ) em parcela sob cobertura morta de Pennisetum purpureum cv. Cameroon, com Gliricidia sepium e solo sem cobertura morta (values of soil moisture in the experimental area (depths of 10 and $20 \mathrm{~cm}$ ) in a plot under mulch of Pennisetum purpureum cv. Cameroon, with Gliricidia sepium and soil without mulch). Seropédica, SIPA, 2008.

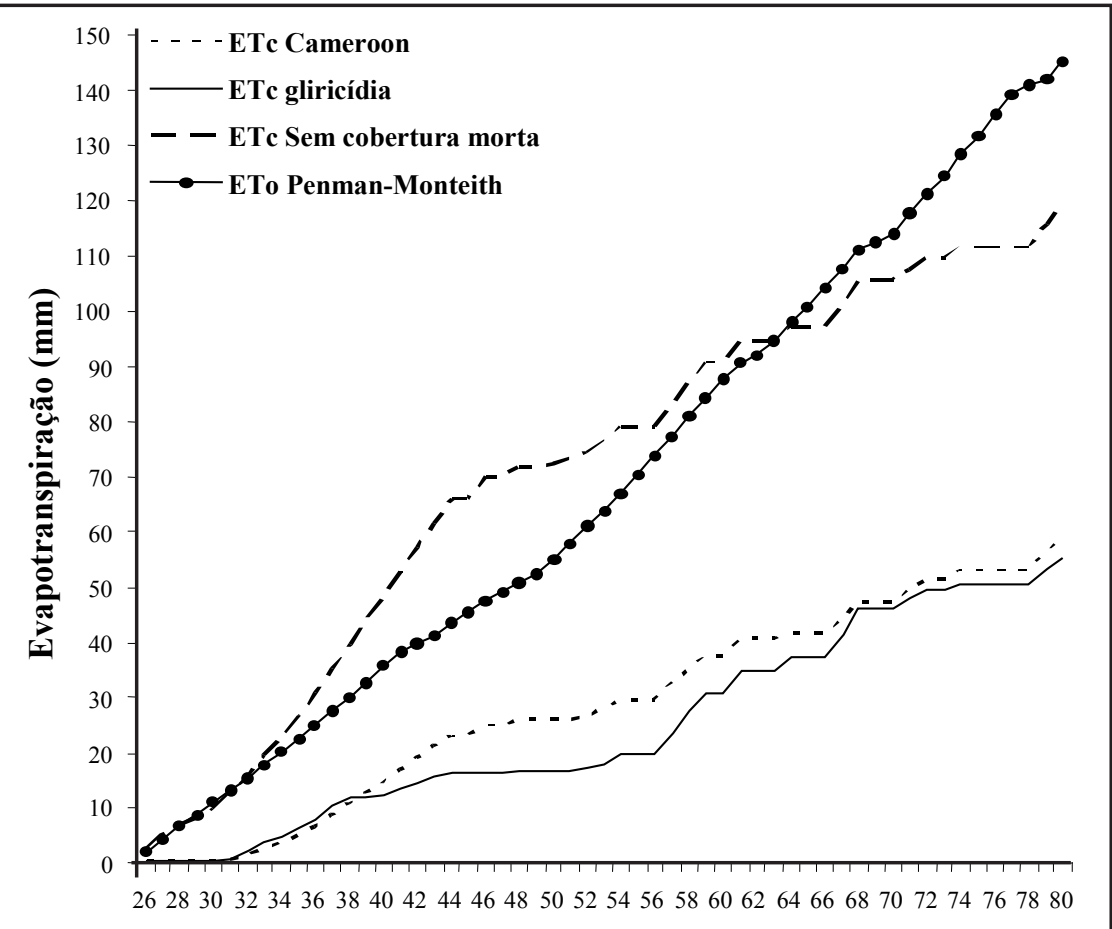

Dias após transplantio

Figura 2. Evapotranspiração acumulada de referência (ETo) e da cultura (ETc), nas diferentes coberturas do solo (com cobertura morta do solo com Pennisetum purpureum cv. Cameroon, com Gliricidia sepium e solo sem cobertura morta), na área experimental (accumulated reference evapotranspiration (ETo) and crop evapotranspiration (ETc) in different soil covers (with mulch of Pennisetum purpureum cv. Cameroon, with Gliricidia sepium and soil without mulch) at the experimental area). Seropédica, SIPA, 2008. mento sem cobertura morta no solo. Isso se deve ao fato da componente evaporação ter se mantido alta em relação à componente transpiração no contexto da evapotranspiração da cultura. Entre os dias 33 e 36, a ETc diária variou de 4,09 a $4,53 \mathrm{~mm}$, diminuindo seus valores a partir desse período.

As umidades relativas média e mínima do ar foram de 50,3 e 15,5\%, respectivamente, combinada com a velocidade média do vento de $1,7 \mathrm{~m} \mathrm{~s}^{-1}$ no dia 36. A ausência de cobertura morta no solo proporcionou maiores perdas por evaporação neste período até que se formou uma faixa efetivamente molhada. A Tabela 2 apresenta os valores médios de kc obtidos neste trabalho e aqueles apresentados por Allen et al. (1998) para a cultura da beterraba de mesa. Nota-se que os valores obtidos para os experimentos com cobertura morta no solo são relativamente próximos aos fornecidos pela FAO, nos estádios iniciais. É nítida a diferença entre os valores de kc obtidos nos experimentos com cobertura morta e sem cobertura do solo. Para as fases inicial, média e final, as diferenças percentuais dos valores de kc para o solo coberto com capim cameroon em relação ao sem cobertura morta foram de 61,$8 ; 33,1$ e $33,3 \%$, respectivamente.

Considerando o experimento com palhada de Gliricídia, essas diferenças foram de 58,8; 35,6 e 27,4\%, respectivamente. Com base nos valores apresentados de variação percentual entre os valores de kc, é possível constatar o efeito da cobertura morta principalmente na fase inicial de cultivo, em que a componente evaporação da água no solo no processo da evapotranspiração torna-se mais relevante. Com o desenvolvimento da cultura, a transpiração torna-se mais relevante, diminuindo essa diferença percentual. Comportamento semelhante foi obtido por Gonçalves et al. (2005) para a cultura da alface, quando as diferenças de consumo de água em solo descoberto em relação ao solo coberto foram de 63,0; 31,8 e $5,2 \%$, respectivamente, para as fases inicial, média e final. Para a cultura da pimenta sob irrigação por gotejamento, Marouelli \& Silva (2007) apresentam os coeficientes $0,40-0,65 ; 0,50 ; 1,00$ e 0,80 para os estádios iniciais, vegetativo, fru- 
tificação e maturação, respectivamente. Para a mesma cultura, Miranda et al. (2006) determinaram para dois ciclos de colheita, valores de kc iguais a 0,3; 1,22 e 0,65 no primeiro ciclo, correspondendo, respectivamente, aos estádios inicial, médio e final, e no segundo ciclo, 1,08 e 0,60 para os estádios médio e final.

A evapotranspiração da cultura da beterraba foi expressivamente inferior (53\% em média) quando foram utilizadas coberturas mortas de capim cameroon ou gliricídia no solo. Os resultados aqui apresentados mostram a importância do uso de coeficientes de cultura locais e adequados ao tipo de manejo do solo adotado, o que contribui para o melhor uso da água de irrigação.

\section{AGRADECIMENTOS}

À Fundação 'Carlos Chagas Filho' de Amparo à Pesquisa do Estado do Rio de Janeiro (FAPERJ) pelo auxílio financeiro; à Coordenação de Aperfeiçoamento de Pessoal de Nível Superior (CAPES), pela bolsa de mestrado concedida ao primeiro autor.

\section{REFERÊNCIAS}

AITA C; GIACOMINI SJ. 2003. Decomposição e liberação de nitrogênio de resíduos culturais de plantas de cobertura de solo solteiras e consorciadas. Revista Brasileira de Ciência do Solo 27: 601-612.

ALLEN RG; PEREIRA LS; RAES D; SMITH M. 1998. Crop evapotranspiration guidelines for computing crop water requirements. Roma: FAO (irrigation and drainage paper 56). 300p.

ALMEIDA MMTB. 2007. Fertilizante de leguminosa: tecnologia inovadora de adubação verde para provisão de nitrogênio em sistema orgânico de produção. Seropédica: UFRRJ. 83p (Tese mestrado).

ALMEIDA MMTB; LIXA AT; SILVA E;
AZEVEDO PHS; DE-POLLI H; RIBEIRO RLD. 2008. Fertilizantes de leguminosas como fontes alternativas de nitrogênio para produção orgânica de alface. Pesquisa Agropecuária Brasileira 43: 675-682.

ANDRADE RS; MOREIRA JAA; STONE LF; CARVALHO JA. 2002. Consumo relativo de água do feijoeiro no plantio direto em função da porcentagem de cobertura morta do solo. Revista Brasileira de Engenharia Agrícola e Ambiental 6: 35-38.

CARVALHO DF; OLIVEIRA NETO DH; RIBEIRO RLD; GUERRA JGM; ROUWS JRC. 2011. Manejo da irrigação associada a coberturas mortas vegetais no cultivo orgânico da beterraba. Engenharia Agrícola 31: 269-277.

CARVALHO DF; SILVA LDB; GUERRA JGM; CRUZ FA; SOUZA AP. 2007. Instalação, calibração e funcionamento de um lisímetro de pesagem. Engenharia Agrícola 27: 363-372.

COSTA RNT; VASCONCELOS JP; SILVA LA; NESS RLL. 2008. Interferência do excesso de água no solo e componentes de produção em beterraba. Horticultura Brasileira 26: 74-77.

DE-POLLI H; ALMEIDA DL. 1988. Manual de Adubação para o Rio de Janeiro. Itaguaí: Ed. Universidade Rural, 179p.

DOORENBOS J; KASSAM AH. 1979. Efeito da água no rendimento das culturas. Rome: FAO. Trad. Gheyi HR; Sousa AA; Damasceno FAV; Medeiros JF. Campina Grande: UFPB (FAO, Estudos de Irrigação e Drenagem, 33), 306p.

ERDEM T; ERDEM Y; ORTA H; OKURSOY H. 2006. Water-yield relationships of potato under different irrigation methods and regimes. Scientia Agricola 63: 226-231.

FILGUEIRA FAR. 2000. Novo Manual de Olericultura: Agrotecnologia moderna na produção e comercialização de hortaliças. Viçosa: UFV. p. 362-366.

GARCIA Y GARCIA A; GUERRA LC; HOOGENBOOM G. 2009. Water use and water use efficiency of sweet corn under different weather conditions and soil moisture regimes. Agricultural Water Management 96: 1369-1376.

GONÇALVES AO; FAGNANI MA; PERES JG. 2005. Efeitos da cobertura do solo com filme de polietileno azul no consumo de água da cultura da alface cultivada em estufa. Engenharia Agricola 25: 622-631.

GUIMARÃES VF; ECHER MM; MINAMI
K. 2002. Métodos de produção de mudas, distribuição de materia seca e produtividade de plantas de beterraba. Horticultura Brasileira 20: 505-509.

HEERMAN DFET. 1985. Et in irrigation management. In: NAT IONAL CONFERENCE ON ADVANCES IN EVAPOTRANSPIRATION, Chicago. Proceedings. St. Joseph: ASAE, p.323-334.

KADAYIFCI A; TUYLU GI; UCAR Y; CAKMAK B. 2005. Crop water use of onion (Allium cepa L.) in Turkey. Agricultural Water Management 72: 59-68.

KELLER J; BLIESNER RD. 1990. Sprinkler and trickle irrigation. New York: Van Nostrand Reinold, 652p.

MANTOVANI EC; BERNARDO S; PALARETTI LF. 2007. Irrigação: principios e métodos. Viçosa: UFV 2a ${ }^{\mathrm{a}}$. ed, p.225-256.

MAROUELLI W; SILVA HR. 2007. Irrigação da pimenteira. Brasília: Embrapa Hortaliças (Circular Técnica 51), $14 \mathrm{p}$.

MEDEIROS JF; SILVA MCC; CÂMARA NETO FG; ALMEIDA AHB; SOUZA JO; NEGREIROS MZ; SOARES SPF. 2006. Crescimento e produção do melão cultivado sob cobertura de solo e diferentes freqüências de irrigação. Revista Brasileira de Engenharia Agrícola e Ambiental 10: 792-797.

MIRANDA FR; GONDIM RS; COSTA CAG. 2006. Evapotranspiration and crop coefficients for tabasco pepper (Capsicum frutescens $\mathrm{L}$.). Agricultural Water Management 82: 237-246.

OLIVEIRA FF; GUERRA JGM; ALMEIDA DL; RIBEIRO RLD; ESPÍNDOLA JAA; RICCI MF; CEDDIA MB. 2008. Avaliação de coberturas mortas em cultura de alface sob manejo orgânico. Horticultura Brasileira 26: $216-220$

SOUZA FILHO JDC; RIBEIRO A; COSTA MH; COHEN JCP. 2005. Mecanismos de controle da variação sazonal da transpiração de uma floresta tropical no nordeste da Amazônia. Acta Amazônica 35: 223-229.

TOPP GC; DAVIS JL; ANNAN AP. 1980. Electromagnetic determination of soil water content: measurements in coaxial transmission lines. Water Resources Research 16: 574-582.

VILLELA ALO. 2007. Variabilidade Espacial da Qualidade Físico-Hídrica do Solo de um Sistema em Produção Agroecológica. Seropédica: UFRRJ. 54p (Tese mestrado). 